\title{
Adult Night Terrors Since Childhood: A Case Report
}

Bailey A Weidner ${ }^{1}$, Nancy A Bergquist ${ }^{2}$ and Tony L Brown ${ }^{3 *}$

${ }^{1}$ University of Colorado-Colorado Springs, USA

${ }^{2}$ Global Neuroscience Initiative Foundation, USA

${ }^{3}$ Columbia University, USA

*Corresponding author: Tony L Brown, Columbia University College of Physicians and Surgeons, USA, Tel: 2679736156; E-mail: tbrown@post.harvard.edu

Received date: November 24, 2015; Accepted date: January 06, 2016; Published date: January 13, 2016

Copyright: @2016 Weidner BA, et al. This is an open-access article distributed under the terms of the Creative Commons Attribution License, which permits unrestricted use, distribution, and reproduction in any medium, provided the original author and source are credited.

\begin{abstract}
Confusion exists among medical professionals in differentiating a night terror episode (NTE) from a nightmare and other sleep disorders in adults. NTEs that persist into adulthood are considered rare in the literature. Devastating injuries occur during NTEs reveal the need for further examination of the physical, mental, and emotional impacts on the patient in order to create lasting curative treatment options. Often, adults who are experiencing NTE do not seek medical help until they hurt themselves, someone else, or until they can no longer cope with the overall effects of disrupted sleep. Much of the literature regarding NTEs is focused on children who, while having a higher prevalence of NTEs, ultimately outgrow the problem without medical intervention. A female with over 40 years of NTE history is studied and the resulting impact on her life is explored. Precipitating factors such as stress and aberrant noise are identified. Various forms of treatment using medications and relaxation techniques are assessed. Misdiagnosis and misunderstanding by medical personnel is also noted in her case. A concise understanding of NTEs throughout all medical specialties is necessary in order to differentiate between the various forms of parasomnias and sleep disorders and to offer appropriate treatment options. Primary care physicians sleep disorder specialists, pain management specialists, orthopaedic and neurology specialists, and psychiatrists could easily encounter such a patient. The importance of recognizing and immediately treating NTEs in adults is vital to the safety and psychology of the possible patients and others affected.
\end{abstract}

Keywords: Night terror; Adult; Somnambulism; Parasomnia; Sleep; Nightmare; SSRI; Benzodiazepine

\section{Introduction}

Night terrors are characterized by the ICD-10-CM code F51.4 as "incomplete arousals from sleep associated with behavior suggesting extreme fright", along with somnambulism, abrupt awakenings, and panicky screams [1]. NTE occur during deep, slow wave, stage iii or iv sleep. In Crisp's article, Sleepwalking/Night Terrors Syndrome in Adults, asserts that children are more affected by NTEs than adults, and men more than women. He also reports common effects of NTEs as exhaustion, injury to self and others, and alcohol dependence; and further, those patients have little to no recall of the episode [2]. Two percent of adults, equating to millions of adults worldwide, suffer from NTEs [3]. Sleep disorders are usually reported secondary to other medical or psychiatric symptoms and are typically assigned a lesser priority of proper diagnosis and treatment [4]. Nightmares occur during rapid eye movement (REM) sleep whereas NTEs occur during non-REM sleep, explaining the unremembered nature of NTE Symptoms during the extended history of NTEs in this case report have had devastating effects on this patient's health. Sources are cited throughout this case report to contrast adult night terror literature in relation to this presenting case.

\section{Case Presentation}

A mid 50's Caucasian female with a 48-year history of night terrors beginning in childhood is studied. Her first memories of NTE start around age five and the episodes have persisted to the present. She was told she had a form of anemia as a child and was diagnosed with iron deficiency anemia during her teens, which coincided with times of fatigue and depressive symptoms. She experienced yearly bronchial infections since childhood and was diagnosed with atypical asthma from second hand cigarette smoke during her childhood.

Symptoms of her NTE include: chronic interrupted sleep, abrupt and panicked sleep arousals, verbal outbursts and screams, a sense of crisis and danger, fright with sudden abrupt movements and leaving her bed, moving throughout her house, and even leaving her house. Originally misdiagnosed as having simple nightmares, she related her symptoms to various medical providers over the years and eventually prescribed zolpidem at $10 \mathrm{mg}$ for 2 months which had no effects on her NTEs. Injuries she has suffered during NTEs include a fractured arm in her teens from jumping out of bed, a herniation of an already bulging disc in her 30 's, and numerous minor injuries from the sudden panicked movements of bounding out of bed and stumbling or tripping on things.

The back injury caused the patient to seek help from a neurologist at a sleep center in the late 1990's who suggested that relaxation techniques before bedtime were as effective as low dose usage of benzodiazepines and thus discouraged a pharmaceutical approach. Since previous counsellors were ill-equipped to treat NTEs, the patient followed the neurologist's advice and tried various relaxation techniques. Muscle contraction sequences, recordings of relaxing sounds, and guided meditation did not change NTE frequency or intensity for her.

Urine neurotransmitter testing was performed ten years ago by a complementary and alternative medicine (CAM) provider and was 
told her level of serotonin was "remarkably low" but she does not recall the exact measurement. No prior baseline testing was performed. Literature suggests a link between low urine serotonin levels and depression [5], coinciding with the reasoning behind supplementing serotonin levels as treatment. She followed a rigorous long-term nutritional supplement program but no lasting change in NTE was noted. The supplements included Metagenics brand: EPA/DHA fish oil at $5 \mathrm{~g} /$ day, multi 4/day, calcium/magnesium minerals, dosage unknown; various brands: 5-HTP oral spray, dosage unknown, taken hourly 8 times/day; DHEA oral spray, dosage unknown, taken BID. Further investigation by her current medical providers include a sleep study, use of selective serotonin reuptake inhibitor (SSRI) medications, use of norepinephrine reuptake inhibitor (NRI) medication, use of diazepam medication, and a sound machine to create a constant level of noise during sleep as foreign noises often trigger NTEs for her. SSRI use: citalopram at $40 \mathrm{mg}$ /day for three months, fluoxetine at $40 \mathrm{mg} /$ day for three months, sertraline at $70 \mathrm{mg} /$ day, and bupropion at 100 $\mathrm{mg} /$ day for six months. The atomoxetine (NRI) dosing is $40 \mathrm{mg} /$ day and diazepam dosing is $5 \mathrm{mg} / \mathrm{night}$, which is the only medication she is currently taking. The sleep study recorded did not record NTEs that night or any apnea-related events. The sound machine helps drown out triggering noises. Diazepam use for the last three months with dosing between 2.5-5 milligrams is tolerable for her and has reduced the frequency of NTE. Stress and anxiety also trigger NTE for her.

The patient in this case is typically unaware of NTE until she is informed by a family member or discovers evidence of sleep-related injury. With gentle comforting interaction from a family member, she can often begin to wake up out of a NTE. Within five to ten minutes she is fully awake and sometimes able to remember the end of the episode. However, the patient often resists these efforts and continues to angrily insist that something is critically wrong and is frustrated that her family member does not understand. The patient is often embarrassed upon full wakefulness and realizing her actions were inappropriate in the absence of any crisis. The patient possesses a striving, driven personality and sometimes experiences. This may coincide with literature that suggests the personality traits of people who experience sleepwalking, night terrors, or nightmares are striving, striving/anxious, and dysphoric, respectively [6]. There is no evidence of NTEs in her children; however, she has witnessed several NTEs in her male sibling and biological father.

\section{Discussion}

While comparing and contrasting somnambulism, night terrors, and nightmares, the clinician must be aware of differing behaviors relating to sleep disorders. People who sleepwalk are often purposeful and clumsy, while people who experience NTEs exhibit fear and people who have nightmares make a rapid shift to wakefulness [7]. The potential for physical harm during a NTE requires proper medical intervention. Medical treatment options for NTEs are commonly benzodiazepine and SSRI medication use. Benzodiazepines affect gamma-aminobutyric acid (GABA), which is the main chemical inhibitor of the central nervous system. GABA receptors in turn control neighbouring chlorine ion channels; both are up-regulated with benzodiazepine use. This hyperpolarizes the cell membrane resulting in the inhibition of action potential firing and less activity. SSRI's prevent reuptake of serotonin in the neuronal synapse. Among the SSRI's, paroxetine has been studied and shown to be effective for some. The 1997 article Adult Night Terrors and Paroxetine by Wilson and Lily-white relayed the effects of various dosages of paroxetine with six adult patients experiencing NTEs since adolescence. All but one patient noticed a recurrence of night terrors upon stopping treatment. Wilson and Lily-white found that paroxetine increased levels of 5hydroxytryptamine $(5-\mathrm{HT})$ within the brainstem by blocking its reuptake. Removal of this treatment caused 5-HT levels to decrease, resulting in increased NTEs. Restarting treatment of paroxetine quickly suppressed NTEs again [8]. Carranza and Dill, in their book Banishing Night Terrors and Nightmares, take issue with the use of drugs in initially treating NTE suggesting an effective psychological approach is lacking. They propose that the use of medication can mask and delay proper treatment through counselling. "Only in the deepest stages of sleep, during the night terror attack, is the sufferer facing the truth about his or her past and present painful emotional realities" [9]. Carranza and Dill also claim physical abuse during early life is the main root cause of NTEs that persist into adulthood. They suggest assistance from a qualified therapist along with concurrent self-help actions will ultimately cure the NTEs. They have studied paroxetine use and suggest it can be helpful at the end of psychotherapy in removing final traces of NTE, instead of at the beginning [7]. The patient in this case study relates potential psychological factors such as a sense of neglect as a child, an alcoholic father and emotionally unavailable mother, no sense of security or safety as a child, and memory blocks of certain time periods during her childhood.

\section{Conclusion}

The unequivocal diagnosis of NTEs is crucial for the safety of patients and those around them. All medical personnel should be educated in the differences among sleep disorders in order to effectively and immediately respond with treatment suggestions. From this original case report, it is concluded that the use of diazepam has been effective in reducing NTE frequency in the last three months. Pursuing deeper psychological counselling is of interest to the patient in this case. This case will primarily have a broad clinical impact across medicine in revealing the need for long term testing of medications and the use of counselling in treating NTE. Significant advancement of medical knowledge should be of priority regarding adult NTEs. Further research utilizing long-term studies can demonstrate the effectiveness of treatment options.

\section{References}

1. http://www.icd10data.com/ICD10CM/Codes/F01-F99/F50-F59/F51-/ F51.4. Accessed 3 Nov 2015.

2. Crisp AH (1996) The Sleepwalking/Night Terrors Syndrome in Adults. Postgrad Med J 72: 599-604.

3. American Academy of Sleep Medicine: Nightmares and Other Disturbing Parasomnias.

4. ICD10Data.com. 2015.

5. Nichkova MI, Huisman H, Wynveen PM, Marc DT, Olson KL, et al. (2012) Evaluation of a novel ELISA for serotonin: urinary serotonin as a potential biomarker for depression. Analytical and Bioanalytical Chemistry 402: 1593-1600.

6. Crisp AH (1996) The Sleepwalking/Night Terrors Syndrome in Adults. Postgrad Med J 72: 599-604.

7. Ibid.

8. Wilson SJ, Lillywhite A (1997) Adult Night Terrors and Paroxetine. Lancet 350: 185.

9. Carranza CR, Dill JR (2004) Banishing Night Terrors and Nightmares. Kensington Publishing Corp, New York. 